\title{
The Multiscale Conformation Evolution of the Financial Time Series
}

\author{
Shupei Huang, ${ }^{1}$ Haizhong An, ${ }^{1,2,3}$ Xiangyun Gao, ${ }^{1}$ Xiaoqing Hao, ${ }^{1}$ and Xuan Huang ${ }^{1}$ \\ ${ }^{1}$ School of Humanities and Economic Management, China University of Geosciences, Beijing 100083, China \\ ${ }^{2}$ Key Laboratory of Carrying Capacity Assessment for Resource and Environment, Ministry of Land and Resources, \\ Beijing 100083, China \\ ${ }^{3}$ Lab of Resources and Environmental Management, China University of Geosciences, Beijing 100083, China
}

Correspondence should be addressed to Haizhong An; ahz369@163.com

Received 29 May 2015; Accepted 22 July 2015

Academic Editor: Michael Small

Copyright (C) 2015 Shupei Huang et al. This is an open access article distributed under the Creative Commons Attribution License, which permits unrestricted use, distribution, and reproduction in any medium, provided the original work is properly cited.

\begin{abstract}
Fluctuations of the nonlinear time series are driven by the traverses of multiscale conformations from one state to another. Aiming to characterize the evolution of multiscale conformations with changes in time and frequency domains, we present an algorithm that combines the wavelet transform and the complex network. Based on defining the multiscale conformation using a set of fluctuation states from different frequency components at each time point rather than the single observable value, we construct the conformational evolution complex network. To illustrate, using data of Shanghai's composition index with daily frequency from 1991 to 2014 as an example, we find that a few major conformations are the main contributors of evolution progress, the whole conformational evolution network has a clustering effect, and there is a turning point when the size of the chain of multiscale conformations is 14 . This work presents a refined perspective into underlying fluctuation features of financial markets.
\end{abstract}

\section{Introduction}

Detecting the dynamical features of a time-dependent complex system mainly depends on time series analysis. This problem is complicated by the nonlinear characteristic of the original time series [1]. Since Lacasa et al. proposed their famous visibility graph algorithm to transfer the time series into networks [2], the last decade has witnessed the success and effectiveness of the complex network in solving nonlinear problems for time series analysis in multiple disciplines, including financial markets [3-5], engineering [6-8], medicine [9-11], and geophysics [12]. Based on these existing contributions, there is yet another trigger of nonlinearity to be concerned with the hidden multiscale information in the frequency domain. It is still a challenge to determine how to transfer a complex network involving multiscale information from the original time series and to explore the underlying evolution features with time and frequency changes simultaneously.

In this paper, we focus on financial time series. As we know, financial markets consist of a number of stakeholders with objects in various time horizons, which results in financial time series comprising a combination of different frequency components [13-15]. Such frequency components form a multiscale conformation behind the original time series and changes within multiscale conformations drive fluctuations of the observed time series values $[16,17]$. In other words, at one time point, a multiscale conformation decides the corresponding observed value. Hence, exploring the dynamic features of the multiscale conformational evolution progress will offer new insight into understanding fluctuations in financial time series from a meticulous perspective.

In regard to the multiscale conformation problem, wavelets offer an effective solution: representing the original time series as a function with two variables, namely, time and frequency [18]. Hence, the implementation of wavelet transform enables us to detect the evolution of different frequency components for different time [19]. In other words, a wavelet working as a "microscope" could observe an original time series using a different "resolution." A finer resolution is better at detecting the details of an original signal, and a low resolution is well-suited for trend analysis [20, 21]. 
Aiming to encode the underlying multiscale conformation evolution features of financial time series, we propose a new algorithm incorporating wavelet transform and the complex network. First, we use the wavelet transform to decompose an original time series into time-frequency domain. We then define the multiscale conformation for one time point with a set of frequency components; a process which offers us a detailed description for current time points rather than for a singular number. Multiscale conformations varying as time changes together form an evolutionary process. We identify the multiscale conformations as nodes, the transmissions over time as edges, and the edges' weight as the frequency of transmission. Hence, we construct the multiscale conformation evolution process as a multiscale evolution complex network. A structural features analysis could help us to explore the underlying dynamical features of financial time series.

\section{Algorithm and Data Description}

2.1. Decomposition in Time-Frequency Domains. First, we use the continuous wavelet transform to obtain the wavelet power spectrum of an original financial time series [22] which could depict the fluctuation of the time series for different frequencies and time [23]. At the heart of continuous wavelet transform is the idea that an original time series should be represented as a function of frequency and time through a wavelet, while the original time series is considered as a function of time alone [18]. A wavelet is a square integral function with real value and zero mean in which there are two parameters: namely, location $(u)$ and scale $(s)$. The location parameter $s$ could determine the wavelet's position in time by shifting the wavelet, while the scale parameter $u$ could stretch or dilate the wavelet to localize different frequencies:

$$
\psi_{u, s}=\frac{1}{\sqrt{s}} \psi\left(\frac{t-u}{s}\right) .
$$

According to the Heisenberg uncertainty principle, there is always a trade-off between the localization of time and scale. For the purpose of extracting features, the Morlet wavelet with $\omega_{0}=6$ is a good choice because it provides a good balance between time and frequency localization [24]:

$$
\psi_{0}(\eta)=\pi^{-1 / 4} e^{i \omega_{0} \eta} e^{-(1 / 2) \eta^{2}}
$$

The continuous wavelet transform could be obtained by projecting the original time series onto the specific wavelet $\psi(\cdot)$ as characterized by location and scale parameters. It could thus be represented as the following equation:

$$
W_{X}(u, s)=\int_{-\infty}^{\infty} \frac{1}{\sqrt{s}} \overline{\psi\left(\frac{t-u}{s}\right) d t}
$$

From the continuous wavelet transform, we can obtain further information about the time series: namely, amplitude. The square of the amplitude $\left|W_{X}\right|^{2}$ is defined as the wavelet power spectrum, which indicates the power distribution of different frequency components of the original time series evolving over time, a large power corresponding to high fluctuation and vice versa. In actuality, the wavelet power result is an $i * j$ matrix $\left\{W_{i j}\right\}$, where $i$ represents a different time point and $j$ represents a different frequency band. We can thus represent the wavelet power matrix as a visible wavelet power spectrum.

\subsection{Constructing the Multiscale Evolution Complex Network}

Step 1 (discretization of the frequency band). Based on wavelet power results, we define the multiscale fluctuation conformation at each time point. The frequency band of the wavelet power matrix ranges from 2 to 512 days, and we discretize the successive frequency bands as sets of 9 separate frequency bands including 2 days, 4 days, 8 days, 16 days, 32 days, 64 days, 128 days, 256 days, and 512 days to represent the multiscale components. The discretized wavelet power matrix is defined as $\left\{W_{i j}^{D}\right\}$.

Step 2 (symbolization of the fluctuation level). According to the actual value of $\left\{W_{i j}^{D}\right\}$, defining the fluctuation level as four types by discretizing a subsequence of continuous observations from one frequency band into four equal zones, the symbolization of the four fluctuation levels is defined as $L_{1}$ (very high), $L_{2}$ (high), $L_{3}$ (weak), and $L_{4}$ (very weak).

Step 3 (definition of a multiscale conformation). Each time point has one corresponding multiscale conformation that consists of nine fluctuation states from nine frequency components. For example, the multiscale conformation for the first time point is $L_{2} L_{2} L_{2} L_{2} L_{3} L_{2} L_{3} L_{2} L_{3}$.

Step 4 (construction of the multiscale evolution complex network). We consider the multiscale conformation for each time point to be a node, transmissions denoted with the corresponding time of multiscale conformations to be edges, and the frequency of the same transmission between conformations to be weight.

2.3. Data Description. We choose the Shanghai (security) composite index (SHCI) from January of 1991 to December of 2014 in daily frequency to serve as a data source. The SHCI represents the fluctuation in the Shanghai stock market comprehensively (Figure 1). There are 5870 data points, and the data are extracted from the wind database. We transform the original SHCI time series into a logarithmic return rate as $X_{t}=\log \left(x_{t} / x_{t-1}\right)$.

\section{Empirical Results}

3.1. Decomposition of the SHCI into Time-Frequency Domain. The wavelet power spectrum of the SHCI can help us to understand the fluctuation of the SHCI as it varies with time and frequency (Figure 2). The horizontal axis depicts time information and the vertical axis displays frequency information. We circle the significant frequency and time intervals with a black line and use warm colors to denote instances in which the SHCI fluctuates highly. It is obvious 


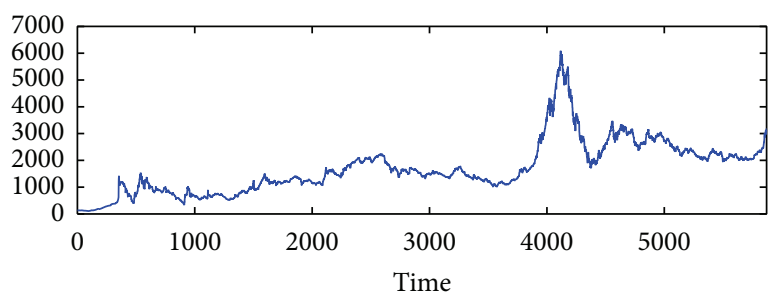

FIGURE 1: Original time series of Shanghai (security) composite index from January 1999 to December 2014.

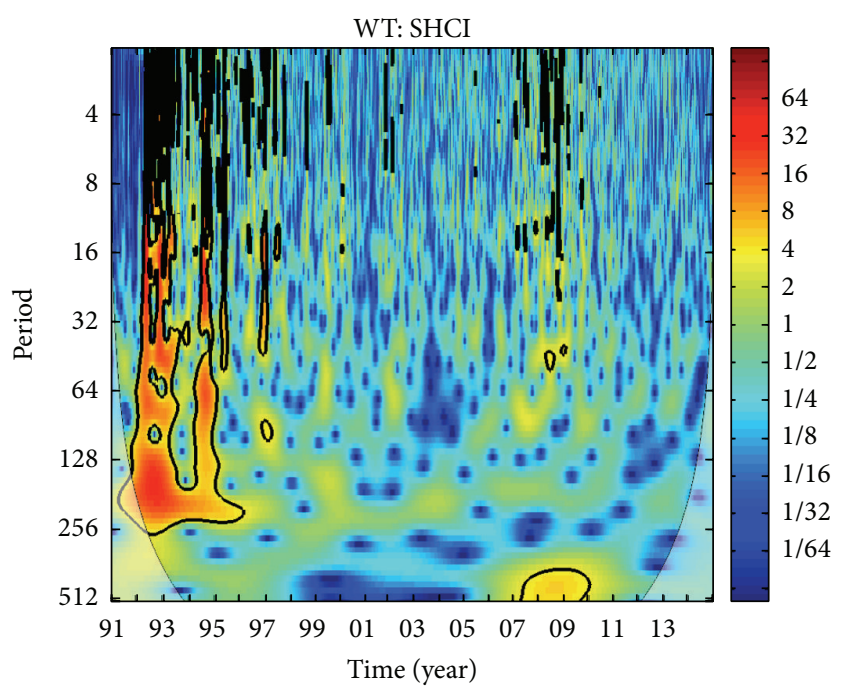

FIgURE 2: The wavelet power spectrum of the SHCI.

that SHCI fluctuates much more highly in the frequency band of 2-256 days from 1992 to 1997 than in other regions.

3.2. Construction of the Multiscale Evolution Networks. Based on the wavelet power spectrum, we obtain 9 frequency bands through discretion (Figure 3). We then normalize discrete wavelet power through a logarithmic transform. A statistical summary demonstrates the value of chosen frequency bands having the maximum of -2.9403 and the minimum of -18.9858 . Hence, we define that there are four fluctuations levels and symbolize them as follows:

$$
\mathrm{fl}_{i}= \begin{cases}L_{1}, & w_{i j}^{D} \in[-2.9403,-6.9517) ; \\ L_{2}, & w_{i j}^{D} \in[-6.9517,-10.9631) ; \\ L_{3}, & w_{i j}^{D} \in[-10.9631,-14.9744) ; \\ L_{4}, & w_{i j}^{D} \in[-14.9744,-18.9558),\end{cases}
$$

According to the above, there are 5870 multiscale conformations for 5870 data points. These multiscale fluctuation conformations transform into each other with changes over time, forming the multiscale fluctuation evolution process. In fact, there are fewer than 5870 types of multiscale conformations due to the repetitive attendance of some conformations. We can thus obtain a directed and weighted multiscale fluctuation evolution network (Figure 4).

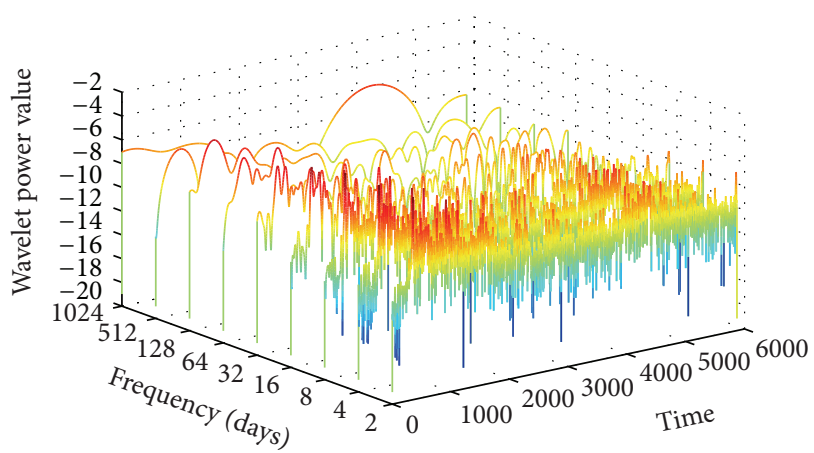

FIGURE 3: The discretion results of the wavelet power spectrum of SHCI.

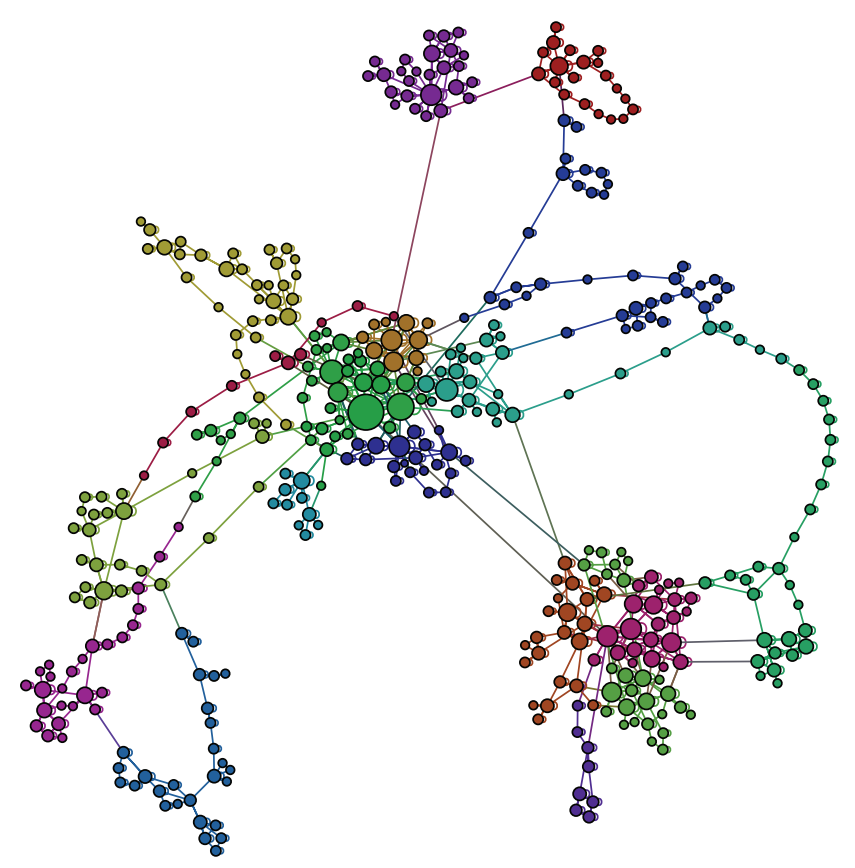

FIgURE 4: The multiscale fluctuation evolution network.

3.3. Evolution Features Analysis. Based on the multiscale fluctuation evolution network, we first use an index of the weighted out-degree to identify the major multiscale conformations. Then, the index of betweenness centralities is used to describe the transmission capability of multiscale conformations. The index of the modularity class could be implemented to explore the clustering effect of multiscale conformations. Finally, we divide the multiscale evolution network into multiscale conformation chains with different lengths and characterize the fluctuation features of SHCI by analyzing the evolution process of these chains.

3.3.1. Major Multiscale Conformations. For the time series containing 5870 time points, there are supposed to be 5870 multiscale conformations and 5869 edges. In fact, due to the repetitiveness of some multiscale conformations, there are 417 nodes and 1165 edges in reality. To identify major multiscale conformations, we introduce the index of the 


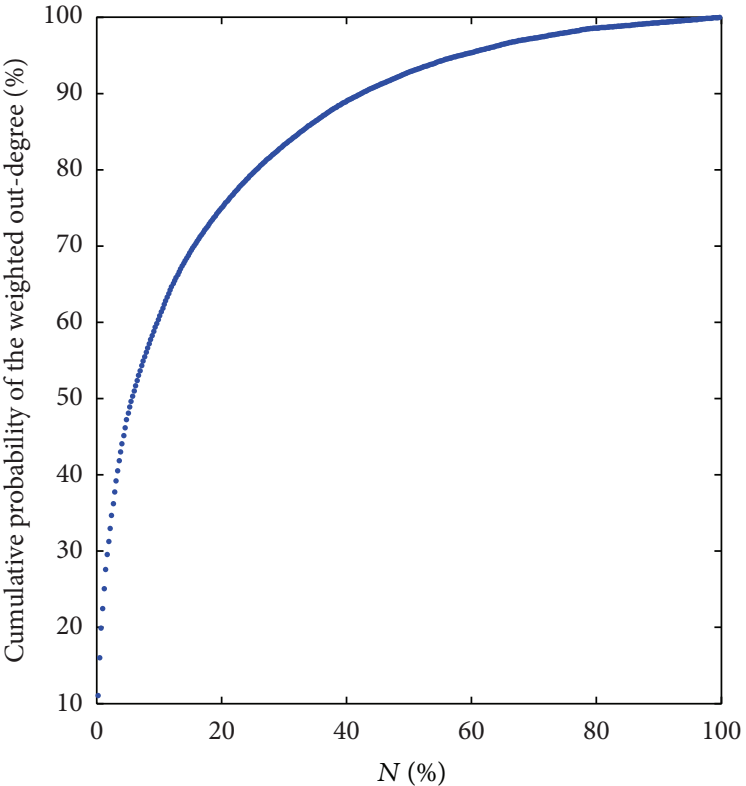

(a)

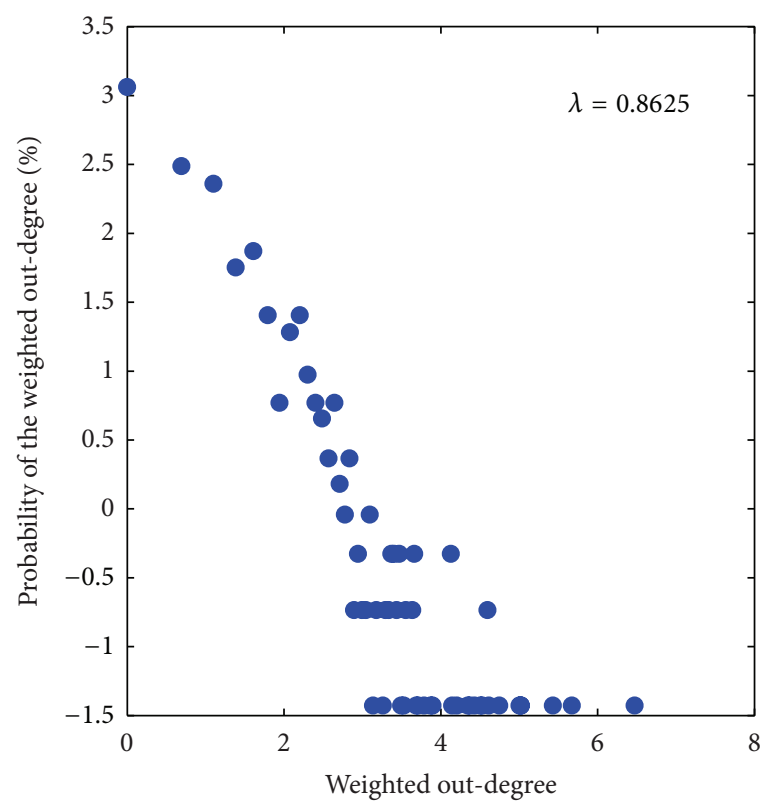

(b)

FIGURE 5: Distributions of the weighted out-degree of node. (a) Cumulative distribution of the weighted out-degree of the node (sorted by the value of the weighted out-degree of the nodes in descending order, $N=1,2, \ldots, 417$ ). (b) Double-logarithmic plot between the weighted out-degree of node and the probability of the weighted out-degree.

weighted out-degree, which not only can depict the number of neighbor conformations of one multiscale conformation but can also demonstrate the weight between multiscale conformations and their neighbors. We define the weight outdegree of one multiscale conformation as follows:

$$
w_{i}^{\text {out }}=\sum_{j \in N_{i}} w_{i j}
$$

where $N_{i}$ represents the number of neighbors shared by the conformation $w_{i}$ and $w_{j}$ represents the weight between conformations $w_{i}$ and $w_{j}$. The weighted out-degree of all multiscale conformations is described in Figure 5(a). We found that $81.09 \%$ of transmissions happen among $26.62 \%$ of multiscale conformations and the distribution of the weighted out-degree of multiscale conformations follows the power law distribution $p\left(w^{\text {out }^{\prime}}\right) \sim w^{\text {out }^{-} \lambda}$ (Figure 5(b)).

The result shows that 111 types of multiscale conformations occur during the evolution process with high frequency and are the main constructors of the SHCI fluctuation status. (Here we list the top 10 conformations in Table 1.)

\subsubsection{Transmission Capability of Multiscale Conformations.} The transmission capability is another crucial characteristic of multiscale conformations during the evolution process and can be described by the index of betweenness centralities. In the complex network of multiscale conformations, the average length of characteristic paths is 11.805 , which means that the average shortest transmission path between any two random multiscale conformations must pass another 11 or 12 conformations. In a small-world network, the shortest transmission path is less than 6 on average and
TABLE 1: The top ten multiscale conformations ranked by the weighted out-degree.

\begin{tabular}{lccc}
\hline Number & Conformations & $\begin{array}{c}\text { Weighted } \\
\text { out-degree }\end{array}$ & $\begin{array}{c}\text { Percentage (\%) } \\
\text { accounts for total } \\
\text { weighted out-degree }\end{array}$ \\
\hline 1 & $L_{3} L_{3} L_{3} L_{3} L_{3} L_{3} L_{3} L_{3} L_{3}$ & 649 & 10.87 \\
2 & $L_{3} L_{3} L_{3} L_{3} L_{3} L_{3} L_{3} L_{3} L_{2}$ & 291 & 4.87 \\
3 & $L_{2} L_{3} L_{3} L_{3} L_{3} L_{3} L_{3} L_{3} L_{3}$ & 228 & 3.82 \\
4 & $L_{3} L_{3} L_{3} L_{3} L_{3} L_{3} L_{3} L_{3} L_{4}$ & 152 & 2.55 \\
5 & $L_{3} L_{3} L_{3} L_{2} L_{3} L_{3} L_{3} L_{3} L_{3}$ & 151 & 2.53 \\
6 & $L_{3} L_{3} L_{2} L_{3} L_{3} L_{3} L_{3} L_{3} L_{3}$ & 149 & 2.50 \\
7 & $L_{3} L_{3} L_{3} L_{3} L_{3} L_{3} L_{3} L_{2} L_{3}$ & 115 & 1.93 \\
8 & $L_{2} L_{3} L_{3} L_{3} L_{3} L_{3} L_{3} L_{3} L_{2}$ & 101 & 1.69 \\
9 & $L_{3} L_{2} L_{3} L_{3} L_{3} L_{3} L_{3} L_{3} L_{3}$ & 99 & 1.66 \\
10 & $L_{3} L_{3} L_{3} L_{3} L_{2} L_{3} L_{3} L_{3} L_{3}$ & 99 & 1.66 \\
\hline
\end{tabular}

the transmission between any two nodes is easy. Hence, in the evolution complex network of multiscale conformations, transmissions between any two multiscale conformations are not convenient and have to be connected by other multiscale conformations that are playing a medium role in the evolution process. Multiscale conformations with high attendance on the shortest transmission path have good transmission capabilities, meaning that these conformations could control more information in the multiscale evolution process. The transmission capability of multiscale conformations can be 
TABLE 2: The weighted out-degree of the top ten conformations ranked by betweenness centrality.

\begin{tabular}{lccc}
\hline Number & Conformations & $\begin{array}{c}\text { Betweenness } \\
\text { centrality }\end{array}$ & $\begin{array}{c}\text { Weighted } \\
\text { out-degree }\end{array}$ \\
\hline 1 & $L_{3} L_{3} L_{3} L_{3} L_{3} L_{3} L_{3} L_{3} L_{3}$ & 0.6445 & 649 \\
2 & $L_{3} L_{3} L_{3} L_{3} L_{3} L_{3} L_{3} L_{3} L_{2}$ & 0.2808 & 291 \\
3 & $L_{3} L_{3} L_{3} L_{3} L_{3} L_{4} L_{4} L_{3} L_{3}$ & 0.2386 & 62 \\
4 & $L_{2} L_{2} L_{2} L_{3} L_{3} L_{2} L_{3} L_{3} L_{3}$ & 0.2382 & 13 \\
5 & $L_{3} L_{3} L_{2} L_{3} L_{3} L_{2} L_{3} L_{3} L_{3}$ & 0.2035 & 39 \\
6 & $L_{3} L_{3} L_{3} L_{3} L_{3} L_{2} L_{3} L_{3} L_{3}$ & 0.1813 & 93 \\
7 & $L_{2} L_{3} L_{3} L_{3} L_{3} L_{3} L_{3} L_{3} L_{4}$ & 0.1787 & 30 \\
8 & $L_{3} L_{3} L_{3} L_{4} L_{3} L_{4} L_{4} L_{3} L_{3}$ & 0.1762 & 19 \\
9 & $L_{3} L_{2} L_{2} L_{3} L_{3} L_{2} L_{3} L_{3} L_{2}$ & 0.1734 & 5 \\
10 & $L_{3} L_{3} L_{3} L_{3} L_{3} L_{3} L_{4} L_{3} L_{3}$ & 0.1649 & 14 \\
\hline
\end{tabular}

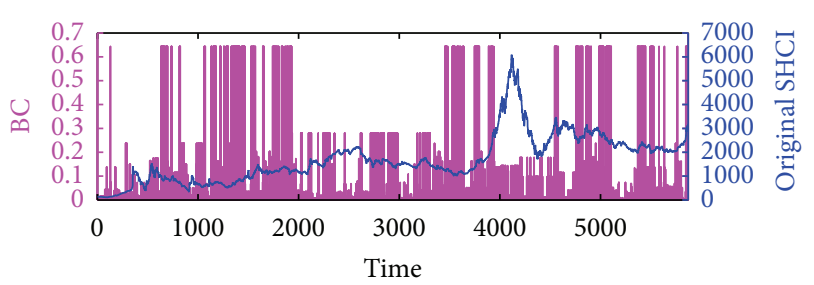

Figure 6: The evolution of the betweenness centrality of multiscale conformations over time.

described by betweenness centralities. The normalized definition of betweenness centralities $\mathrm{BC}_{i}$ of conformation $i$ can be written as follows:

$$
\mathrm{BC}_{i}=\frac{\sum_{j}^{k} \sum_{k}^{n} q_{j k}(i) / q_{j k}}{n^{2}-3 n+2}, \quad j \neq k \neq i, j<k
$$

where $q_{j k}$ is the total number of shortest paths between nodes $j$ and $k . q_{j k}(i)$ is the number of shortest paths between nodes $j$ and $k$ that pass node $i$.

The evolution of the betweenness centrality of multiscale conformations over time is depicted in Figure 6. Conformations with high betweenness centrality have a clustering effect caused by either the repetitiveness of a single conformation with high betweenness centrality or frequent transmissions among conformations with high betweenness centrality. We identify top 10 multiscale conformations with high betweenness centrality (Table 2). Conformations with high betweenness centrality work like a bridge to link the other conformations in the multiscale complex network. Their appearances are the necessary condition for transmission among conformations that do not connect directly. In some cases, without these conformations with high betweenness centrality, the transmission among some specific conformations will be impossible. Among all multiscale fluctuation conformations with high betweenness centrality, those that also have high weighted out-degree play a dominant role in the evolution complex network, while other conformations with low weighted out-degree may be in a temporary transition status. For example, the multiscale conformation

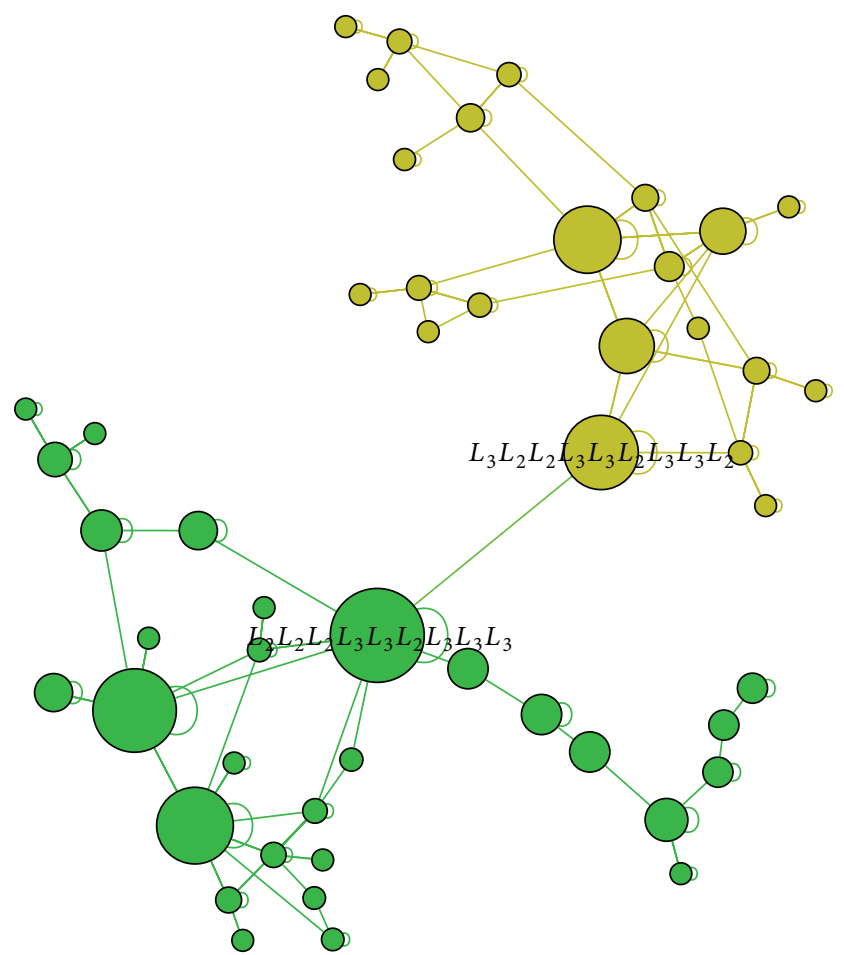

FIgURE 7: An example of multiscale conformations with high betweenness centrality and low weighted out-degree. The multiscale conformations $L_{3} L_{2} L_{2} L_{3} L_{3} L_{2} L_{3} L_{3} L_{2}$ and $L_{2} L_{2} L_{2} L_{3} L_{3} L_{2} L_{3} L_{3} L_{3}$ have betweenness centrality of 0.1734 and 0.2382 and weighted outdegree of 5 and 13, respectively. They link two communities and their appearance may indicate that the transform moves between these two communities.

named $L_{3} L_{2} L_{2} L_{3} L_{3} L_{2} L_{3} L_{3} L_{2}$ and $L_{2} L_{2} L_{2} L_{3} L_{3} L_{2} L_{3} L_{3} L_{3}$ with high betweenness centrality and low weighted outdegree links two communities, the appearance of which may indicate the transform moves from one community to another (Figure 7).

3.3.3. The Clustering Effect. Based on weighted out-degree and betweenness centrality, we find that transmissions among some multiscale conformations are difficult. Without some conformations as medium, all multiscale conformations will be separated into several distinct groups. We ask whether there are a number of multiscale conformations clustered closely that could easily transmit to each other. Therefore, we use a modularity index to measure the partition of communities in the evolution network of multiscale conformations, with higher values of the modularity demonstrating a better partition of a complex network. The definition of modularity can be written as follows [25]:

$$
Q=\frac{1}{2 m} \sum_{i, j}\left[w_{i, j}-\frac{A_{i} A_{j}}{2 m}\right] \delta\left(c_{i}, c_{j}\right),
$$

where $w_{i, j}$ is the weight of the edge between nodes $i$ and $j$. $A_{i}=\sum_{j} w_{i, j}$ is the sum of the weights of the edges that connected with node $i . c_{i}$ is the community that node $i$ is 
assigned to. $\delta\left(c_{i}, c_{j}\right)$ is 1 when $c_{i}=c_{j}$ and is 0 otherwise $m=(1 / 2) \sum_{i, j} w_{i, j}$.

The modularity partitions algorithm is divided into two phases repeated iteratively. First, each node is considered to be a community. The number of communities is therefore equal to that of the nodes. We then evaluate the gain of the modularity $(\Delta Q)$. Node $i$ will be moved to a neighboring community only when the gain of the modularity is positive; otherwise node $i$ will remain in the contemporary community. This phase will be repeated until no individual move can improve modularity. The gain of the modularity $(\Delta Q)$ can be calculated by

$$
\begin{aligned}
\Delta Q= & {\left[\frac{\sum C_{\text {in }}+A_{i, \text { in }}}{2 m}-\left(\frac{\sum \text { tot }+A_{i}}{2 m}\right)\right] } \\
& -\left[\frac{\sum \text { in }}{2 m}-\left(\frac{\sum \text { tot }}{2 m}\right)^{2}-\left(\frac{A_{i}}{2 m}\right)^{2}\right],
\end{aligned}
$$

where $\sum C_{\mathrm{in}}$ is the sum of the weights of all edges inside community $C, \sum$ tot is the sum of the weights of edges incident to all nodes inside community $C$. $A_{i}$ is the sum of the weights of the edges incident to $i . A_{i, m}$ is the sum of the weights of the edges from node $i$ to all nodes in community $C$, and $m$ is the sum of the weights of all edges in the network.

In the second phase, taking the communities found in the first phase to be nodes, we can build up a new network. The weight of the edges of the new network can be obtained from the sum of the weights of edges between nodes in two corresponding communities. Edges between nodes of the same community lead to self-loops in the new network. When the second phase is finished, the first phase is repeated, thus resulting in a new network. These two phases are repeated until there are no more changes (details in [25]).

We apply the algorithm to the evolution complex network of multiscale conformations. The modularity of the evolution complex network is 0.809 and the whole complex network is separated into 20 communities. The number of each community ranges from 8 to 32 . Inside each community, multiscale conformations transmit to each other continently. Among these communities, some cannot transmit to another directly. In this case, conformations with high betweenness centrality work as pivots to connect isolated communities.

3.3.4. The Transmission Capability of Multiscale Conformation Chains. The evolution complex network of multiscale conformations is characterized by a clustering effect. Furthermore, we divided the evolution process of multiscale conformations into various chains that consist of different numbers of multiscale conformations. The number of multiscale conformations inside the chain is defined as the size of the chain. We then explore the transmission features of these chains with size changes. We define the chain size as $n$ and observe the whole multiscale fluctuation process from a sliding perspective. Hence, the number of multiscale conformations sets will be $5870-n+1$.

First, the number of multiscale conformation chains increases with increasing size, meaning that the diversity of

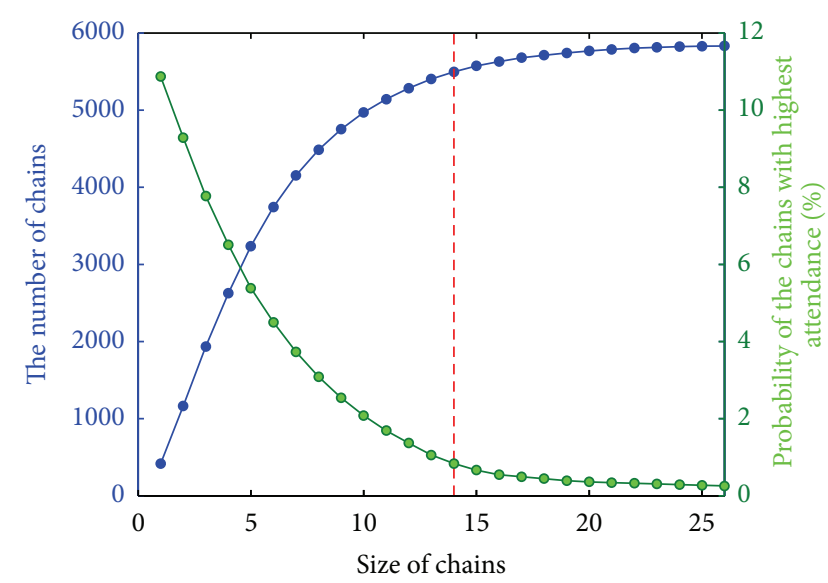

FIGURE 8: The number of chains of multiscale conformations and percentage of the chains with the highest attendance accounts in the total number of chains of multiscale conformations.

chains is growing when their components increase. Simultaneously, the percentage of chains with the highest attendance accounts in the total number of chains decreases as the size of the chain grows. As mentioned above, it is obvious that there is a turning point at which the repetition of chains is scarce. For a multiscale complex network, the turning point appears when the size of the chain becomes 14 .

Another point deserving notice is the status of chains with the highest attendance. Generally, the self-transmission of multiscale conformations with high weighted out-degree constitutes the chain of the multiscale fluctuation with high attendance. Hence, when the multiscale conformation with high weighted out-degree appears for several days, this conformation may last for a period in the near future (Figure 8).

\section{Discussion and Conclusions}

The nonlinearity of financial time series caused by the hidden multiscale information complicates the exploration of underlying mechanisms. We present a multiscale algorithm combining wavelet transform and the complex network, offering meticulous insight into characterizing the evolution process of the multiscale conformation that drives the fluctuation of observed values within the original time series. We first implement a continuous wavelet to obtain the wavelet power spectrum of the original time series that depicts its fluctuation as changes with time and fluctuation. We then define the multiscale conformations of each time point based on the construction of an evolution complex network. Finally, we characterize the evolution complex network through analyses of the major conformations, the transmission capacity of the conformations, the clustering effect, and the transmission of the chains of multiscale conformations.

We use the SHCI daily data from January 1990 to December 2014 as an example and construct the evolution complex network of multiscale conformations. More specifically, the weighted out-degree and betweenness centrality of the multiscale conformations follow the power law distribution. 


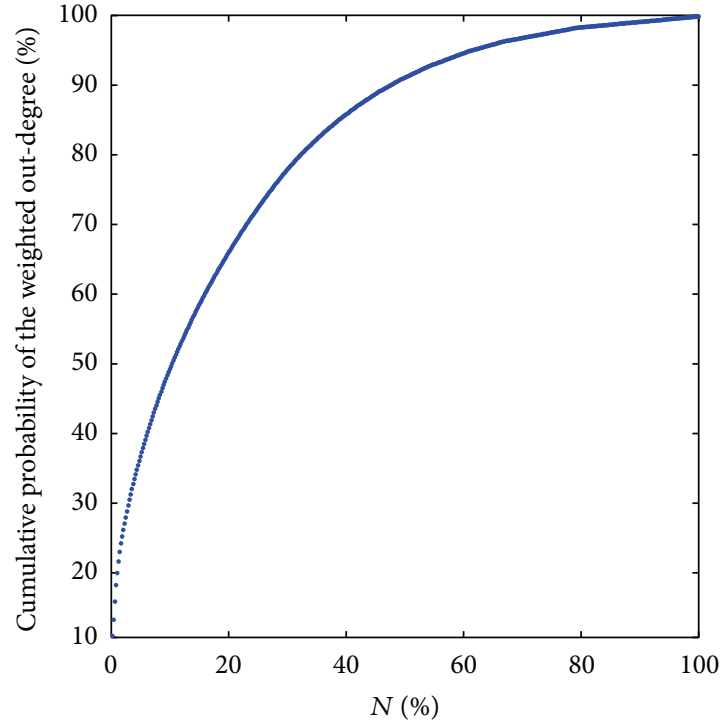

(a) DAX 30

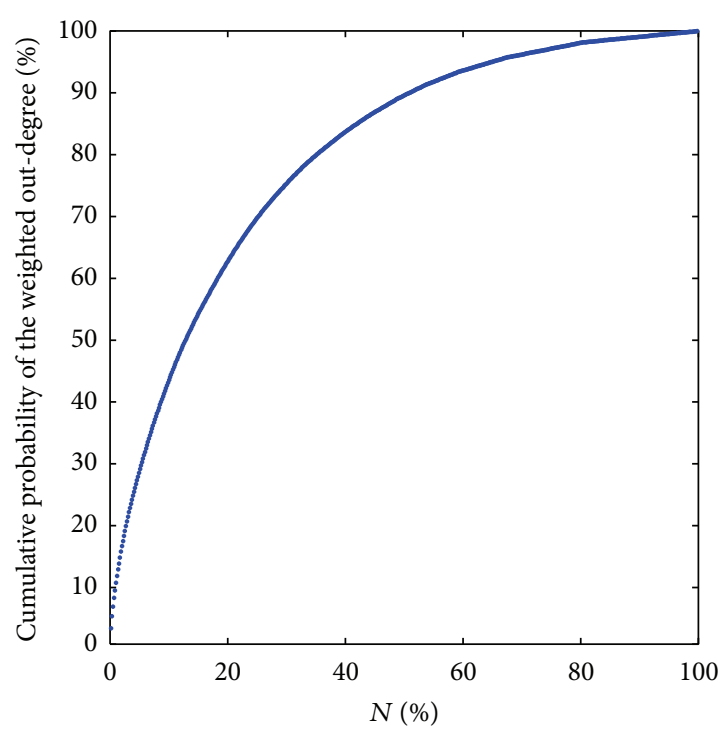

(c) FTSE100

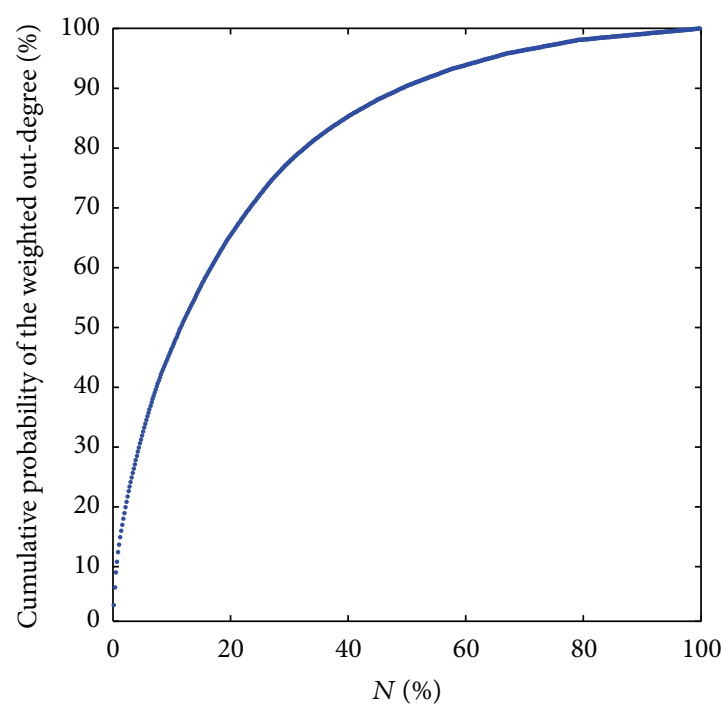

(e) DJIA

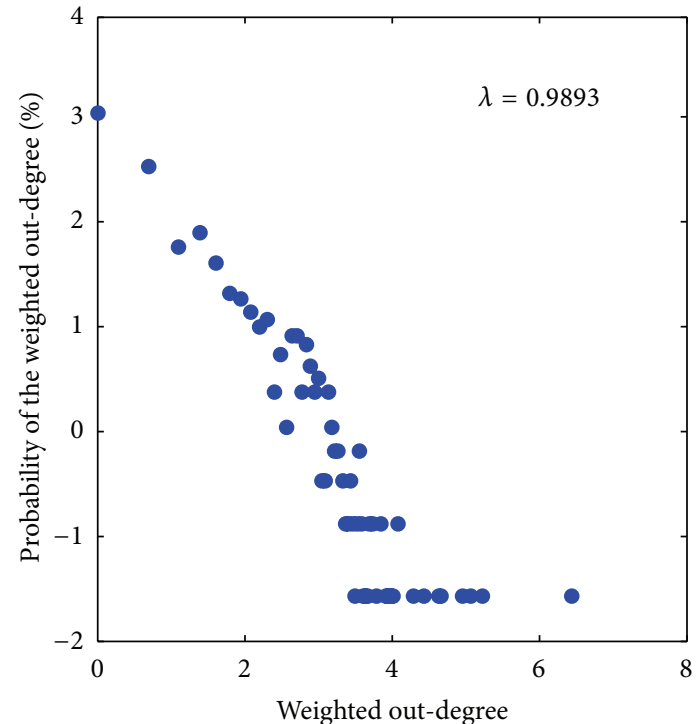

(b) DAX 30

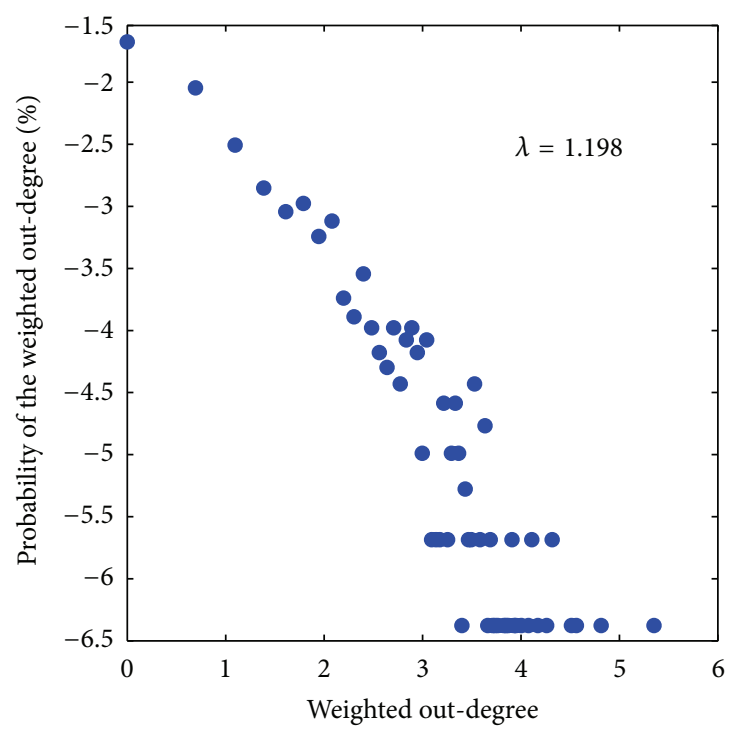

(d) FTSE100

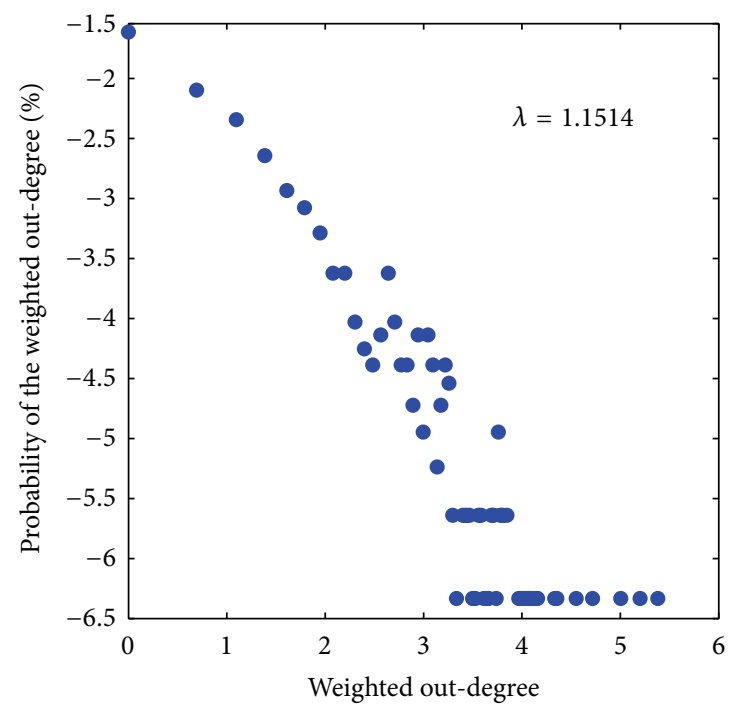

(f) DJIA

FIgure 9: Continued. 


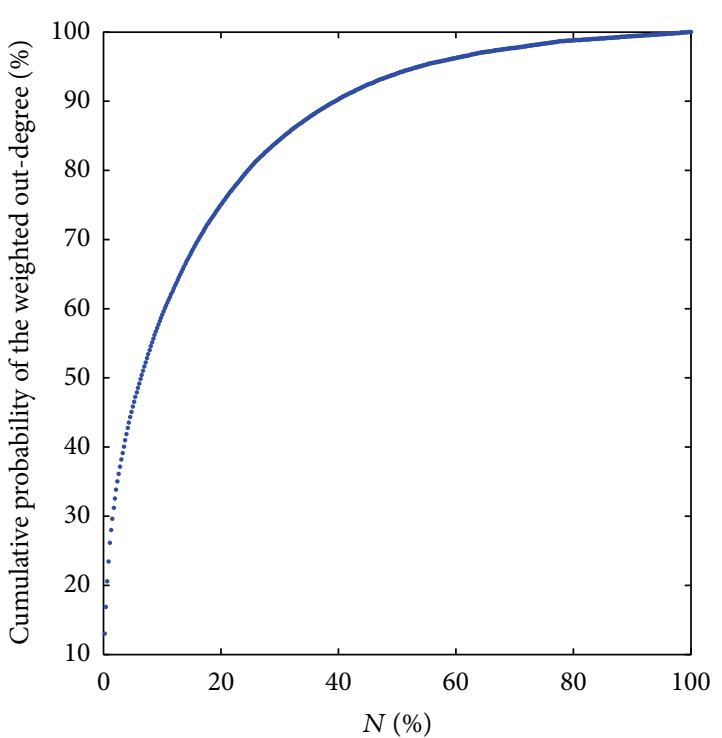

(g) NIKKEI225

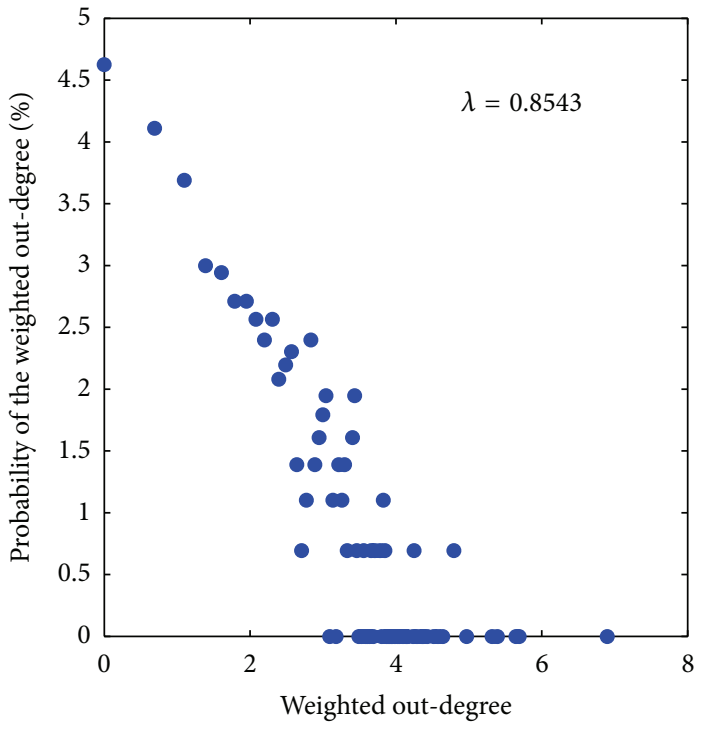

(h) NIKKEI225

FIGURE 9: Distributions of the weighted out-degree of node. Cumulative distribution of the weighted out-degree of the node of (a) DAX, (c) FTSE100, (e) DJIA, and (g) NIKKEI225 (sorted by the value of the weighted out-degree of the nodes in descending order). Double-logarithmic plot between the weighted out-degree of node and the probability of the weighted out-degree of (b) DAX, (d) FTSE100, (f) DJIA, and (h) NIKKEI225.

We could therefore identify major conformations that are the main contributors in the whole network and major transmit conformations which serve as pivots connecting separated conformations. Such information could be considered to be a reference to changes within the SHCI. We then find that the whole evolution complex network can be partitioned into 20 communities inside of which transmissions among conformations are easily made. Among some communities the major transmit conformations only transmit to each other. In this case, the appearance of transmit conformations could be a signal that depicts structural changes characteristic of SHCI. Finally, through the analysis of transmissions of multiscale fluctuation chains, the turning point of SHCI appears when chain size is 14 .

In addition, to prove the validation and effectiveness of the algorithm, we apply it to other stock indices from four major countries, namely, UK (FTSE100), Germany (Dax 30), US (DJIA), and Japan (NIKKEI225), from January of 1991 to December of 2014 in daily frequency. They contain 6084, 6254,6079 , and 5912 data points, respectively. We found that the weighted out-degrees of these four stock indices also follow the power law distribution and their exponents of the distributions of the weighted out-degree are approximately 1. Moreover, it is obvious that nearly $80 \%$ transmissions happen among roughly $30 \%$ of the multiscale conformations. These results prove that the multiscale evolution features of the four major stock indices are very similar to that of the SHIC (Figure 9).

In this study, we try to put forward an approach to uncovering the underlying mechanism of nonlinearity expressed by a financial time series, which can help us to understand financial markets in greater detail. However, the nonlinearity of financial prices may be affected by other factors that we try to involve in a future study.

\section{Conflict of Interests}

The authors declare that there is no conflict of interests regarding the publication of this paper.

\section{Acknowledgments}

This research was partly supported by the National Natural Science Foundation of China (Grant no. 71173199) and the Fundamental Research Funds for the Central Universities (Grant no. 35732015060).

\section{References}

[1] P. C. Ivanov, M. G. Rosenblum, C. K. Peng et al., "Scaling behaviour of heartbeat intervals obtained by wavelet-based time-series analysis," Nature, vol. 383, no. 6598, pp. 323-327, 1996.

[2] L. Lacasa, B. Luque, F. Ballesteros, J. Luque, and J. C. Nuno, "From time series to complex networks: the visibility graph," Proceedings of the National Academy of Sciences the United States of America, vol. 105, no. 13, pp. 4972-4975, 2008.

[3] X. Y. Gao, H. Z. An, W. Fang, X. Huang, H. Li, and W. Q. Zhong, "Characteristics of the transmission of autoregressive sub-patterns in financial time series," Scientific Reports, vol. 4, article 6290, 2014.

[4] X. Y. Gao, H. Z. An, W. Fang et al., "Transmission of linear regression patterns between time series: from relationship in time series to complex networks," Physical Review E, vol. 90, no. 1, Article ID 012818, 2014. 
[5] X. Y. Gao, H. Z. An, W. Fang, H. J. Li, and X. Q. Sun, "The transmission of fluctuant patterns of the forex burden based on international crude oil prices," Energy, vol. 73, pp. 380-386, 2014.

[6] Z.-K. Gao and N.-D. Jin, "A directed weighted complex network for characterizing chaotic dynamics from time series," Nonlinear Analysis: Real World Applications, vol. 13, no. 2, pp. 947-952, 2012.

[7] Z..-K. Gao, X.-W. Zhang, N.-D. Jin, R. V. Donner, N. Marwan, and J. Kurths, "Recurrence networks from multivariate signals for uncovering dynamic transitions of horizontal oil-water stratified flows," EPL, vol. 103, no. 5, Article ID 50004, 2013.

[8] Z. K. Gao, M. S. Ding, H. Geng, and N. D. Jin, "Multivariate multiscale entropy analysis of horizontal oil-water two-phase flow," Physica A: Statistical Mechanics and Its Applications, vol. 417, pp. 7-17, 2015.

[9] M. Zanin, P. Sousa, D. Papo et al., "Optimizing functional network representation of multivariate time series," Scientific Reports, vol. 2, article 630, 2012.

[10] E. Siggiridou, D. Kugiumtzis, and V. Kimiskidis, "Correlation networks for identifying changes in brain connectivity during epileptiform discharges and transcranial magnetic stimulation," Sensors, vol. 14, no. 7, pp. 12585-12597, 2014.

[11] K. Lehnertz, G. Ansmann, S. Bialonski, H. Dickten, C. Geier, and S. Porz, "Evolving networks in the human epileptic brain," Physica D: Nonlinear Phenomena, vol. 267, pp. 7-15, 2014.

[12] M. Paluš, D. Hartman, J. Hlinka, and M. Vejmelka, "Discerning connectivity from dynamics in climate networks," Nonlinear Processes in Geophysics, vol. 18, no. 5, pp. 751-763, 2011.

[13] L. Vacha and J. Barunik, "Co-movement of energy commodities revisited: evidence from wavelet coherence analysis," Energy Economics, vol. 34, no. 1, pp. 241-247, 2012.

[14] X. Huang, H. An, X. Gao, X. Hao, and P. Liu, "Multiresolution transmission of the correlation modes between bivariate time series based on complex network theory," Physica A: Statistical Mechanics and Its Applications, vol. 428, pp. 493-506, 2015.

[15] X. Jia, H. An, W. Fang, X. Sun, and X. Huang, "How do correlations of crude oil prices co-move? A grey correlationbased wavelet perspective," Energy Economics, vol. 49, pp. 588598, 2015.

[16] C. B. Li, H. Yang, and T. Komatsuzaki, "Multiscale complex network of protein conformational fluctuations in single-molecule time series," Proceedings of the National Academy of Sciences of the United States of America, vol. 105, no. 2, pp. 536-541, 2008.

[17] J. Yu, Y. Lei, and H. An, "Dynamic features of China's crude oil price based on multi-scale entropy," Resources \& Industries, vol. 14, no. 6, pp. 31-39, 2012.

[18] I. Daubechies, Ten Lectures on Wavelets, SIAM, 1992.

[19] C. Torrence and G. P. Compo, "A practical guide to wavelet analysis," Bulletin of the American Meteorological Society, vol. 79, no. 1, pp. 61-78, 1998.

[20] S. G. Mallat, "Multiresolution approximations and wavelet orthonormal bases of $\mathrm{L}^{2}(\mathrm{R})$," Transactions of the American Mathematical Society, vol. 315, no. 1, pp. 69-87, 1989.

[21] S. G. Mallat, "A theory for multiresolution signal decomposition: the wavelet representation," IEEE Transactions on Pattern Analysis and Machine Intelligence, vol. 11, no. 7, pp. 674-693, 1989.

[22] D. Maraun and J. Kurths, "Cross wavelet analysis: significance testing and pitfalls," Nonlinear Processes in Geophysics, vol. 11, no. 4, pp. 505-514, 2004.
[23] A. Grinsted, J. C. Moore, and S. Jevrejeva, "Application of the cross wavelet transform and wavelet coherence to geophysical time series," Nonlinear Processes in Geophysics, vol. 11, no. 5/6, pp. 561-566, 2004.

[24] A. Rua and L. C. Nunes, "International comovement of stock market returns: a wavelet analysis," Journal of Empirical Finance, vol. 16, no. 4, pp. 632-639, 2009.

[25] V. D. Blondel, J. L. Guillaume, R. Lambiotte, and E. Lefebvre, "Fast unfolding of communities in large networks," Journal of Statistical Mechanics: Theory and Experiment, vol. 2008, Article ID P10008, 2008. 


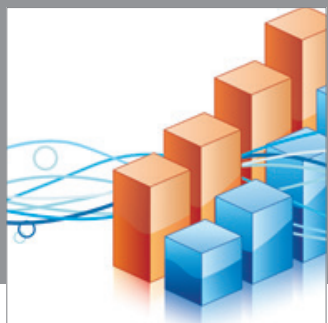

Advances in

Operations Research

mansans

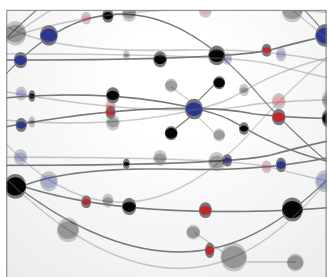

The Scientific World Journal
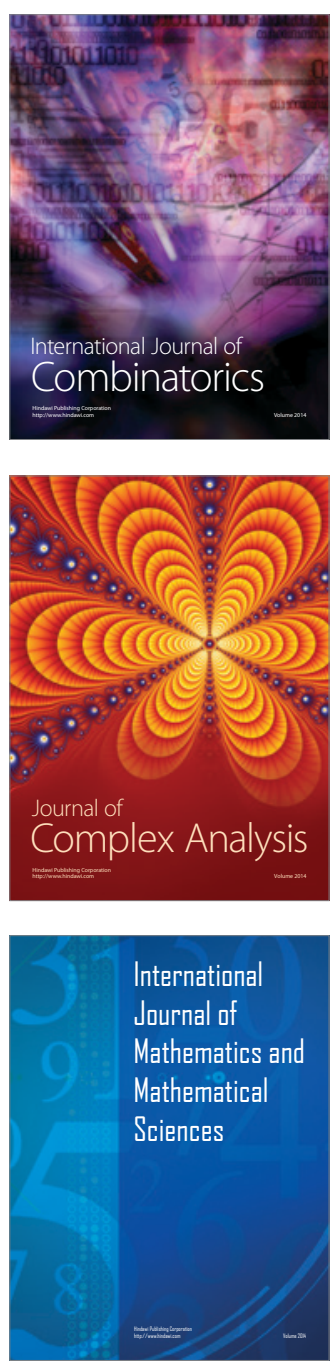
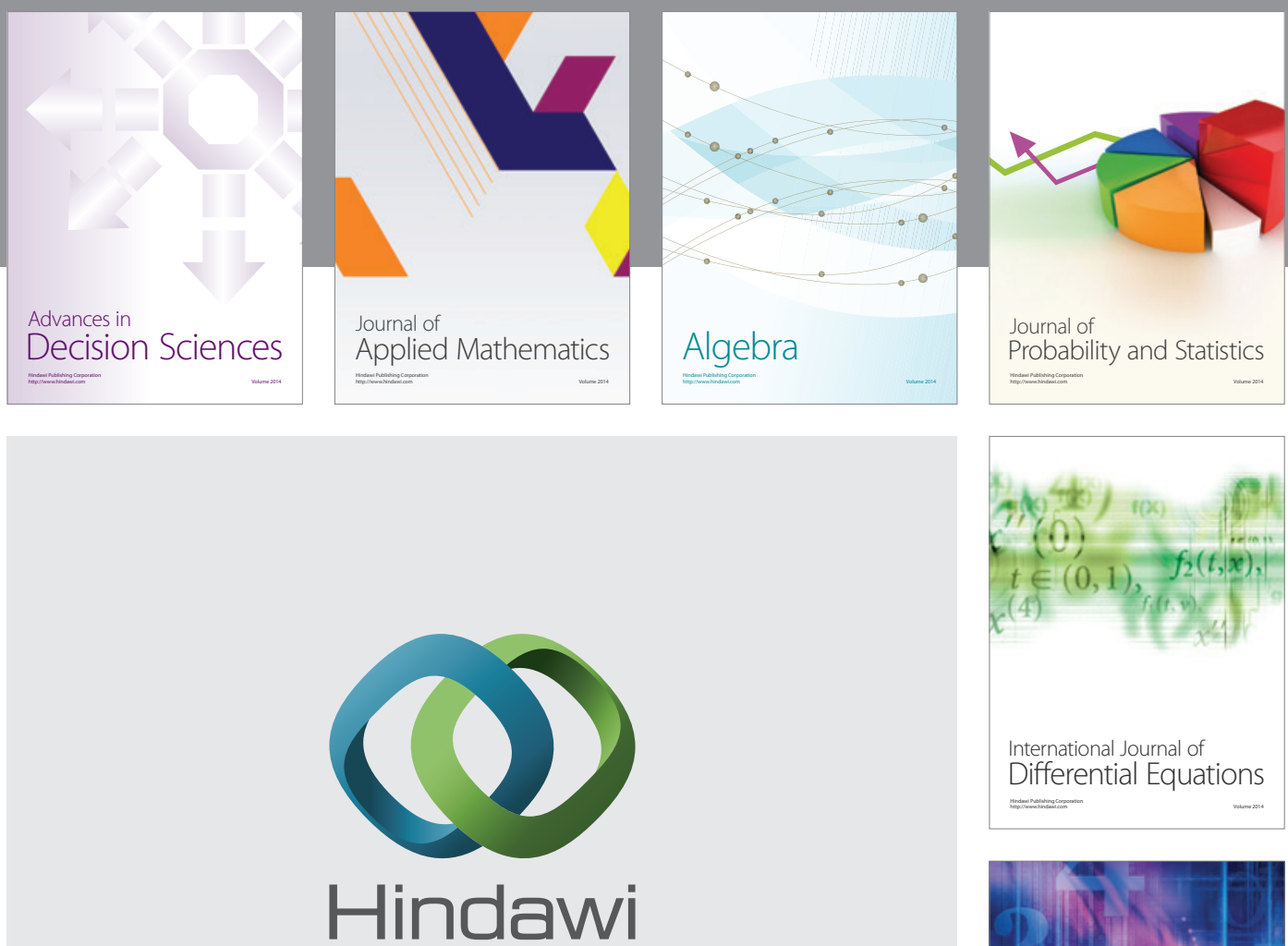

Submit your manuscripts at http://www.hindawi.com
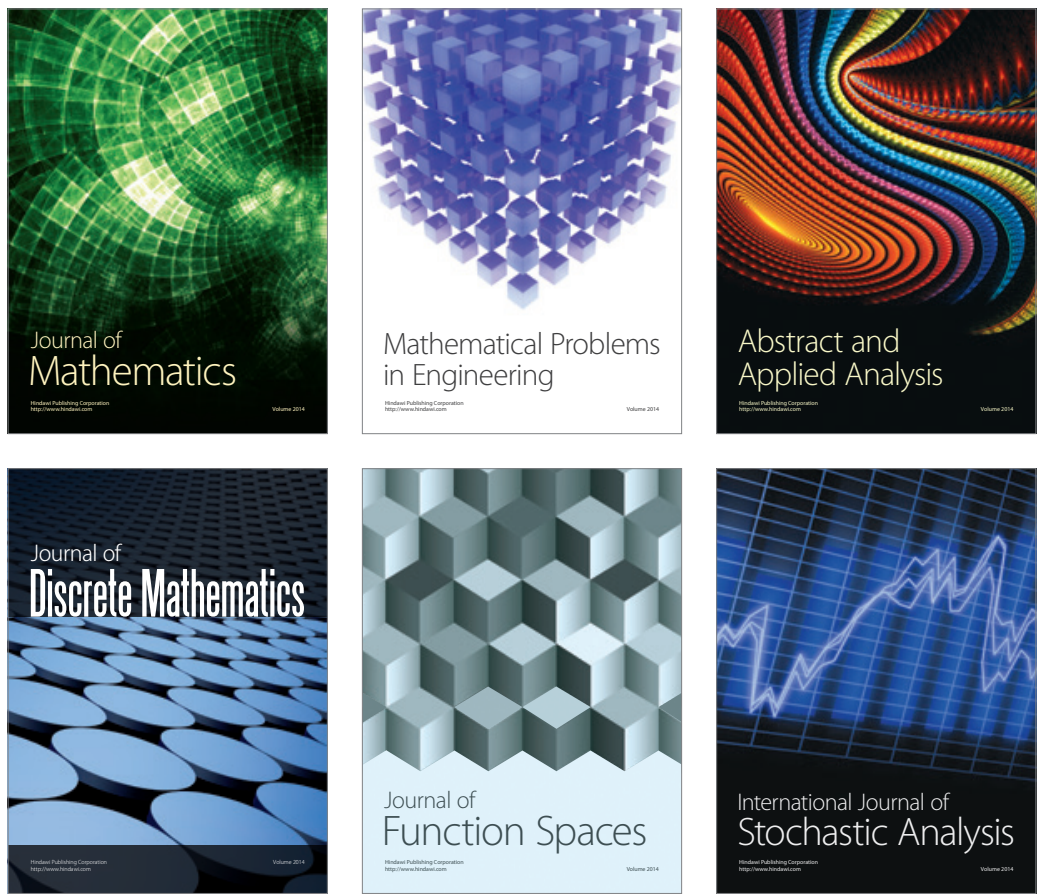

Journal of

Function Spaces

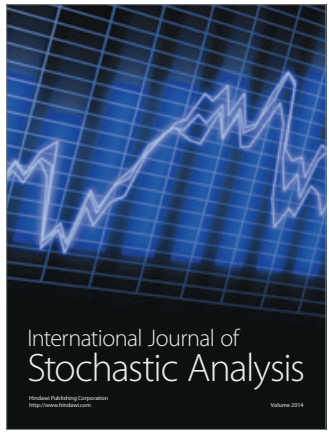

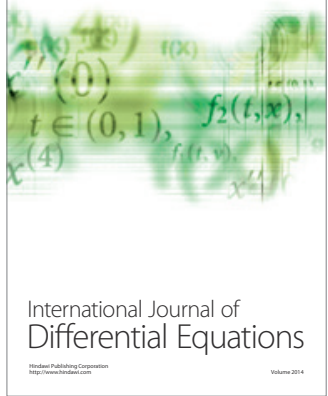
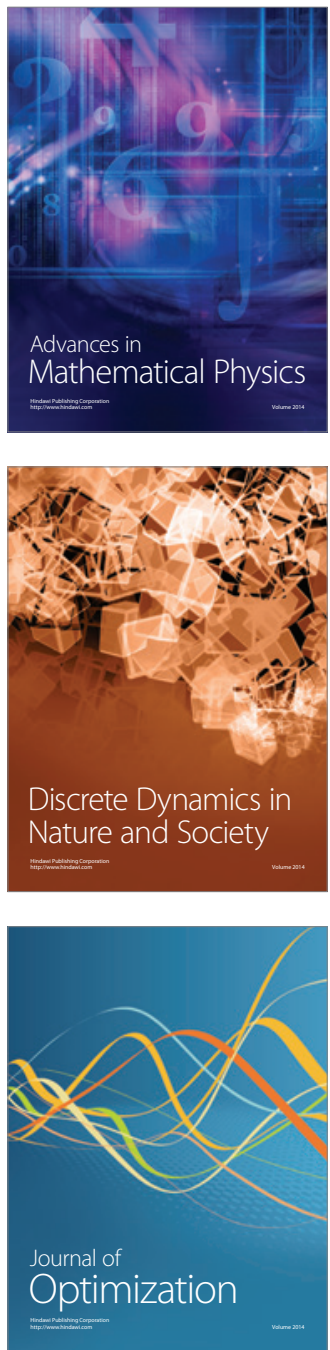